\title{
Quantitative Evaluation of Filter Aided Sample Preparation (FASP) and Multienzyme Digestion FASP Protocols
}

\author{
Jacek R. Wiśniewski*
}

Biochemical Proteomics Group, Department of Proteomics and Signal Transduction, Max-Planck-Institute of Biochemistry, Am Klopferspitz 18, D-82152 Martinsried, Germany

\section{Supporting Information}

ABSTRACT: Filter aided sample preparation (FASP) and related methods gain increasing popularity for proteomic sample preparation. Nevertheless, the originally published FASP method has been criticized by several authors, who reported low digestion performance. In this work, we reevaluate FASP and the related multienzyme digestion (MED) FASP method. We use different types of animal tissues and cultured cells and test the performance of the method under various conditions. We analyze the protein to peptide conversion by assessing the yield of peptides, frequency of peptides with missed cleavage sites, and the reproducibility of FASP. We identify conditions allowing efficient protein processing with high peptide yields and demonstrate advantages of the two step digestion strategy over single step digestion with trypsin. In addition, we show that FASP outperforms in-solution cleavage strategies. Our results clearly demonstrate that the performance of digestion varies between different types of samples. We show that MED FASP in combination with the total protein approach provides highly reproducible protein abundance values. The presented data can be used as a guide for optimization of sample processing.
$S_{\mathrm{w}}^{\mathrm{an}}$ mple preparation is the most critical step in the proteomic workflow. In shotgun bottom-up proteomics, which is the most popular way to analyze proteomes, a large number of different methods for protein extraction and digestion coexists. Besides the original "gel-" and "in-solution" digestion protocols, approaches using solid phase supports ${ }^{1,2}$ or reaction vessels, considered as proteomic reactors, ${ }^{3-9}$ gain increasing popularity. The latter class also includes ultrafiltration based methods that employ centrifugal protein concentrators. These "reactors" allow sample purification, chemical derivatization, and enzymatic digestion. They enable more flexibility in sample processing, such as successive cleavage, ${ }^{10,11}$ and sample fractionation, ${ }^{12}$ which increases depth of proteomics analysis ${ }^{10}$ and facilitates identification of low abundant proteins. ${ }^{11}$ An additional advantage of the ultrafiltration approaches is the purity of the digests, which is a prerequisite for effective peptide fractionation and mass spectrometry using $\mathrm{MuDPIT}^{13}$ or isoelectric focusing.

Filter aided sample preparation (FASP) and the multiple enzyme digestion FASP (MED FASP) method were developed and successfully applied to the analysis of cultured cells as well as tissue lysates from fresh, frozen, and formalin fixed, paraffin embedded samples. Across different studies using various sample type and sample size, we have reported $50-70 \%$ yields of the protein to peptide conversion and low content of peptides with missing cleavages. ${ }^{12,14-16}$ However, performance of FASP was not tested systematically. In this report, we evaluate the performance of FASP and MED FASP applied to processing of whole lysates samples from mouse organs and human cells. We test the digestion efficiency in terms of quality and yields of peptides using different conditions and sample amounts. We show also the advantages of the ultrafiltration based digestion protocol compared to in-solution digestion methods.

\section{EXPERIMENTAL SECTION}

Tissue Isolation and Lysis. Liver, brain, and skeletal muscles were dissected from Balb C57BL/6 mice. Liver microsomes were prepared from fresh mouse liver by centrifugation of the postnuclear supernatant at $21000 \mathrm{~g}$ for 1 h. Mouse tissues were homogenized in $0.1 \mathrm{M}$ Tris- $\mathrm{HCl}, \mathrm{pH} 7.8$, 0.05 M DTT using a T10 basic Ultraturrax dispenser (IKA, Staufen). The ratio of tissue to the buffer was 1:5-1:10 (g/ $\mathrm{mL})$. After addition of SDS to a final concentration of $2 \%(\mathrm{w} /$ $\mathrm{v})$, the homogenates were sonicated in a Branson type instrument, Sonifier 250 (Heinemann, Schwäbisch Gmünd), operating at $20 \%$ duty cycle and 3-4 output for $1 \mathrm{~min}$, and were then incubated in a boiling-water-bath for $5 \mathrm{~min}$. After cooling to room temperature, lysates were clarified by centrifugation at $16000 \mathrm{~g}$ for $10 \mathrm{~min}$. Cultured cells and liver microsomes were lysed in $0.1 \mathrm{M}$ Tris- $\mathrm{HCl}, \mathrm{pH} 7.8,0.05 \mathrm{M}$

Received: March 4, 2016

Accepted: April 27, 2016

Published: April 27, 2016 
$\mathrm{DTT}$, and the lysate was sonicated and incubated at $100{ }^{\circ} \mathrm{C}$ for 5 min.

Protein Digestion Using FASP and MED FASP Methods. Protein lysates were processed by the FASP $^{5}$ and MED FASP protocol ${ }^{10}$ using Microcon 30k centrifugal ultrafiltration units (Merck, Darmstadt) operated at $10000 \mathrm{~g}$. We reduced the centrifugation force below the maximum recommended by manufacturer $(14000 g)$. This was necessary to circumvent an often observed damage of the ultrafiltration membrane during centrifugation. Aliquots containing 25-400 $\mu \mathrm{g}$ of total protein were mixed with $200 \mu \mathrm{L}$ of $8 \mathrm{M}$ urea in 0.1 $\mathrm{M}$ Tris/ $\mathrm{HCl}, \mathrm{pH} 8.5$ (UA), in the ultrafiltration unit and then centrifuged at $20{ }^{\circ} \mathrm{C}$, for $15 \mathrm{~min}$. The eluates were discarded; $100 \mu \mathrm{L}$ of UA was pipetted into the filtration unit, and the units were centrifuged again. Then, $50 \mu \mathrm{L}$ of $0.05 \mathrm{M}$ iodoacetamide in UA was added to the filters, and samples were incubated in darkness for $20 \mathrm{~min}$. Filters were washed twice with $100 \mu \mathrm{L}$ of UA followed by two washes with $100 \mu \mathrm{L}$ of $0.05 \mathrm{M}$ Tris $/ \mathrm{HCl}$, pH 8.5 (digestion buffer DB), containing $0,1,2,3,4,6$, or $8 \mathrm{M}$ urea and were digested in $40 \mu \mathrm{L}$ of the buffer used for washing at $37{ }^{\circ} \mathrm{C}$ for $18 \mathrm{~h}$, using endoproteinase LysC (MED FASP) or trypsin (FASP), at an enzyme to protein ratio of $1: 100$. The released peptides were collected by centrifugation at $10000 \mathrm{~g}$ for $10 \mathrm{~min}$ followed by two washes with $100 \mu \mathrm{L}$ of DB. In the MED FASP protocol, the material remaining on the filter was digested with trypsin using the above conditions, except that the cleavage reaction was performed only for $2 \mathrm{~h}$. The eluates containing $10 \mu \mathrm{g}$ of total peptide were desalted on $\mathrm{C}_{18}$ StageTips ${ }^{17}$ and concentrated to a volume of $4-5 \mu \mathrm{L}$ and were stored frozen at $-20{ }^{\circ} \mathrm{C}$ until mass spectrometric analysis.

In-Solution Digestion. Twenty $\mu \mathrm{L}$ aliquots of SDS lysates containing $100 \mu \mathrm{g}$ of total protein were mixed with $80 \mu \mathrm{L}$ of absolute ethanol and incubated at $-80{ }^{\circ} \mathrm{C}$ for $3 \mathrm{~h}$. The precipitate was collected by centrifugation at $10000 \mathrm{~g}$ for 10 min. The resulting pellet was washed with $80 \%$ ethanol, vacuum-dried, and dissolved in $25 \mu \mathrm{L}$ of UA. Then, $1 \mu \mathrm{g}$ of LysC was added, and the mixture was incubated at $37{ }^{\circ} \mathrm{C}$ overnight. After dilution with $75 \mu \mathrm{L}$ of $\mathrm{DB}$, the samples were digested with $1 \mu \mathrm{g}$ of trypsin at $37{ }^{\circ} \mathrm{C}$ for $3 \mathrm{~h}$. For determination of the digestion efficiency, the digest was passed through the Microcon 30k to isolate peptides.

Determination of Total Protein and Peptide Contents. Total protein and total peptide contents in the lysates were determined by the tryptophan fluorescence assay (WF assay) in the microtiter plate format ${ }^{18}$ using Corning Costar 96-well black flat bottom polystyrene plates (Sigma-Aldrich, Taufkirchen). Tryptophan was used as standard.

LC-MS/MS Analysis. Analysis of the peptide mixtures was performed with an LTQ Orbitrap instrument (Thermo-Fisher Scientific) as described previously. ${ }^{19,20}$ Briefly, aliquots containing $\sim 5 \mu \mathrm{g}$ of peptides were injected and separated on a reverse phase column $(20 \mathrm{~cm} \times 75 \mu \mathrm{m}$ inner diameter $)$ packed with $1.8 \mu \mathrm{m}$ C18 particles (Dr. Maisch $\mathrm{GmbH}$, Ammerbuch-Entringen, DE) using a $4 \mathrm{~h}$ acetonitrile gradient in $0.1 \%$ formic acid at a flow rate of $250 \mathrm{~nL} / \mathrm{min}$. The $\mathrm{LC}$ was coupled to the mass spectrometer via a nanoelectrospray source (Proxeon Biosystems, now Thermo Fisher Scientific). The LTQ Orbitrap was operated in data dependent mode with survey scans acquired at a resolution of 60000 at $\mathrm{m} / z$ 400. For CID fragmentation, up to the 10 most abundant precursor ions from the survey scan with charge $\geq+2$ within the $300-1700 \mathrm{~m} /$ $z$ range were selected. The normalized collision energy was 35 .
The dynamic exclusion parameters were $90 \mathrm{~s}$ and $5 \mathrm{ppm}$. The $\mathrm{MS}^{2}$ spectra were acquired in the ion-trap.

Data Analysis. The MS data from mouse and human material were analyzed in MaxQuant software. ${ }^{21}$ Proteins were identified by searching MS and MS/MS data of peptides against a decoy version of the UniProtKB (May 2013). Carbamidomethylation of cysteines was set as a fixed modification. Nterminal acetylation and oxidation of methionine were set as variable modifications. Up to two missed cleavages were allowed. The initial allowed mass deviation of the precursor ion was up to $6 \mathrm{ppm}$, and for the fragment masses, it was $0.5 \mathrm{Da}$. The "match between runs" option was enabled to match identifications across samples within a time window of $2 \mathrm{~min}$ of the aligned retention times. The maximum false peptide discovery rate was specified as 0.01 . Protein concentrations were calculated by the TPA method. ${ }^{14}$ The statistical analysis of the data sets was performed in SigmaPlot12 software. The percentage of partially cleaved peptides (MCPs) was normalized by their spectral intensities.

\section{RESULTS AND DISCUSSION}

FASP protocols that allow quantitative depletion of SDS and sample digestion were developed several years ago. Since then, the protocols have been optimized for sample preparation efficiency and workload. First, substitution of the $10 \mathrm{kD}$ cutoff Microcon filtration units with $30 \mathrm{kDa}$ cutoff units reduced the time needed for the multiple centrifugation steps $^{22}$ by approximately 3 -fold. Second, by decreasing of the centrifugation force from $14000 \mathrm{~g}$ to $10000 \mathrm{~g}$, we eliminated losses of sample due to frequently observed breaking of the filtration membrane (unpublished). Since the frequency of filter breaking has been varying between batches of filters, we attribute the reduction of the ultrafiltration membrane robustness variation in the manufacturing process rather than an effect of a prolonged storage. Third, we reduced the number of solutions used in the procedure to only two: a "cleaning solution" (UA) and a "digestion buffer" (DB). Finally, we found that there are limits in the sample amounts that can be processed at peptide yield and that addition of urea to the digestion mixture has only negligible influence on the FASP efficiency. Moreover, we found that overall performance of the methods depends on the sample type. These observations were derived from several unrelated studies, rendering an overall assessment difficult. Here, we have benchmarked FASP performance systematically.

Capacity of the Filtration Units Is Limited. To test the capacity of the filtration device, we processed different amounts of sample using LysC and trypsin in either FASP or MED FASP formats (Figure 1). Whole tissue lysates of mouse liver and brain were used. The highest yields of the protein to peptide conversion were observed for samples containing up to $100 \mu \mathrm{g}$ of total protein. For higher loads, a continuous decrease of yields was observed. For loads of $400 \mu \mathrm{g}$ of total protein, the peptide yield was halved compared to the $100 \mu \mathrm{g}$ loads. Similar results were obtained using filtration units with vertically oriented membranes (Figure 1A). However, for sample amounts of $100 \mu \mathrm{g}$ of total protein or less, the peptide yields were considerably reduced compared to the flat bottomed units.

Presence of Denaturant Has a Negligible Effect on the Digestion Efficiency. Urea is a commonly used reagent in various proteomic sample preparation protocols. In the FASP methods, concentrated urea is used for depletion of SDS, and in the original protocol sample, digestion was carried out in $8 \mathrm{M}$ 

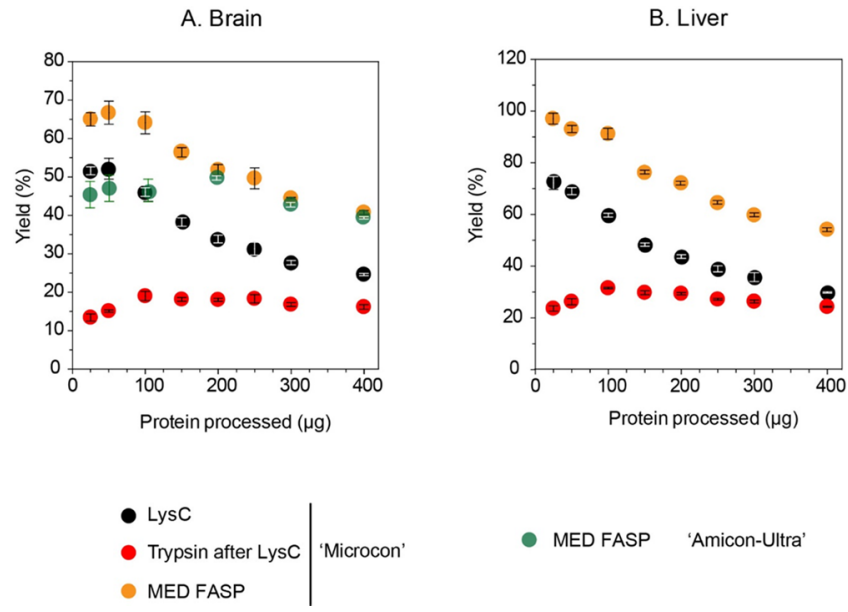

- MED FASP 'Amicon-Ultra'

Figure 1. Efficiency of protein to peptide conversion at varying amounts of processed total protein. Whole tissue lysates of mouse brain (A) and liver (B) in 2\% SDS were processed by MED FASP. Digestions with LysC and trypsin were performed in the absence of urea. The yields were expressed as a ratio of the amount of eluted peptides to total protein amount processed. Black and red dots refer to the first and the second digestion in MED FASP, respectively. The orange dots (MED FASP) are the sum of the yields from both digestions. The ratio of LysC and trypsin to total protein was 1:100. The experiments were performed in triplicates. Bars indicate standard deviation.

urea solution. In this study, to test the effect of urea on the digestion efficiency, we processed whole lysates from mouse brain, mouse muscle, and human $\mathrm{CaCo}-2$ cells using buffers containing 0 to $8 \mathrm{M}$ off the denaturant (Figure 2). In the FASP
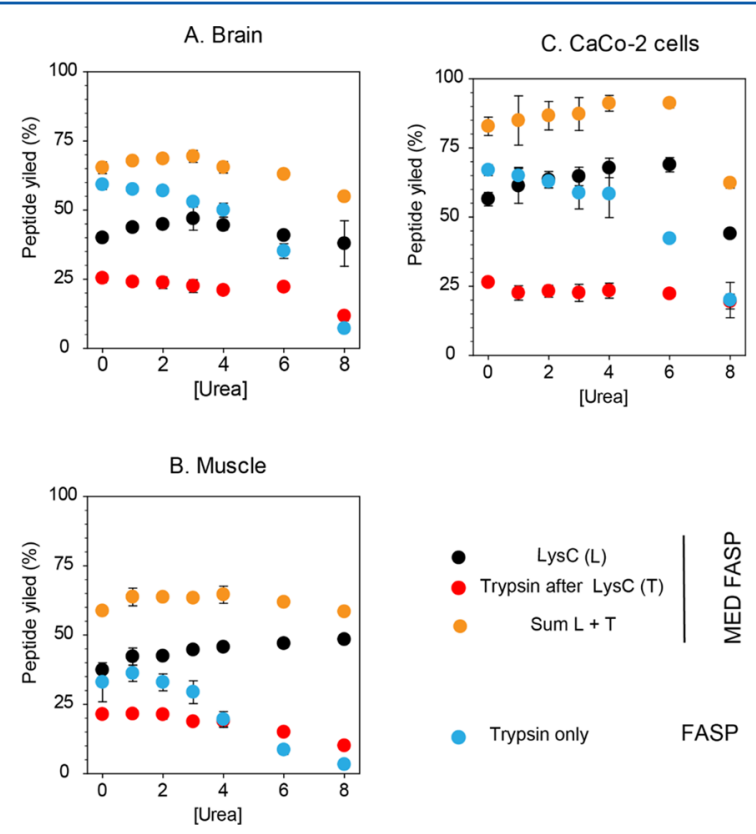

Figure 2. Efficiency of protein to peptide conversion in the absence or presence of urea. $100 \mu \mathrm{g}$ aliquots of whole lysates of mouse brain (A) and muscle (B) and human CaCo-2 cells in $2 \%$ SDS were processed by FASP (blue dots) and MED FASP (orange dots). The yields were expressed as a ratio of the amount of eluted peptides to total protein amount processed. Black and red dots refer to the first and the second digestion in MED FASP, respectively. The orange dots are the sum of the yields from both digestions. procure, using single step digestion with trypsin, in the presence of 1 or $3 \mathrm{M}$ urea, had only minimal effect on the peptide yield. At urea concentrations exceeding $4 \mathrm{M}$, the peptide yields were strongly reduced. A similar relation of urea concentration and digestion yields was observed in both mouse tissues and $\mathrm{CaCo}-2$ lysates. In the MED FASP format, the increasing concentrations of urea were accompanied by a maximal 20\% increase of peptide yields during the LysC cleavage. In parallel, the yields of trypsin digestion were decreasing with an increase of urea concentration. Overall, the sum of the peptides released by LysC and trypsin was changing only a little between 0 and $6 \mathrm{M}$ urea.

In general, MED FASP resulted in a greater peptide amount compared to FASP. We observed clear differences in the peptide yields between tissues and cell samples. Proteins in the lysate of a cultured cells were mostly susceptible to digestion, resulting in up $90 \%$ yields with MED FASP. In contrast, digestion of muscle proteins resulted in $60-65 \%$ yields with MED FASP. Single tryptic digestion of this sample type resulted at the best in $\sim 30 \%$ yield.

Digestion in Urea Does Not Improve the Sample Performance in LC-MS/MS Analysis. The peptide mixtures obtained by FASP and MED FASP from brain, muscle, and CaCo- 2 cells were analyzed by LC-MS/MS using $4 \mathrm{~h}$ gradient chromatography and an "Orbitrap" mass spectrometer. This analysis revealed that the presence of urea in concentrations of 4 or $6 \mathrm{M}$ used during digestion has little effect on the sequencing efficiency of peptides. As a consequence, the number of identified peptides varied only little (Figure 3). However, we observed significant differences between different types of samples. The peptide mixtures obtained from the cultured cells and brain were more frequently sequenced than the peptides from muscle, and this was reflected in the numbers of identified peptides. The $\mathrm{LysC}$ and tryptic fractions from MED FASP analyzed separately resulted in fewer peptide identifications than the tryptic peptide fraction generated by FASP. However, when the numbers of unique peptides were summed, their total number increased up to $90 \%$ compared to the single tryptic digests. The larger number of unique peptides results in identification of an additional $30-40 \%$ proteins (Figure 3C,F,I).

Presence of Urea Increases the Rate of Peptides with Missed Cleavages. Enzymatic protein cleavage is always accompanied by generation of partially digested peptides. The rate of their occurrence was subject to recent studies. ${ }^{23,24}$ Both papers showed that digestion with trypsin results in a high content of partially digested peptides. "In-solution" tryptic digests of yeast and bacterial extracts contained $28 \%$ and $46 \%$ of peptides with missed cleavages (MCPs), respectively. ${ }^{23,24}$ Combining tryptic digestion with LysC predigestion reduced the extent of missed cleavages to $19 \%$.

Figure 4 shows rates of MCPs found in the analysis of the tissue and cell samples. Across all samples and conditions used, digestion with LysC resulted in the lowest frequency of MCPs ranging from $2 \%$ to $4 \%$, in the absence or the presence of urea up to $6 \mathrm{M}$. At $8 \mathrm{M}$ urea, the rate of missed cleavages increased to $10 \%$ in the muscle sample (Figure $4 \mathrm{C}$ ). The second step in the MED FASP procedure, the digestion with trypsin, resulted in $5-10 \%$ of MCPs in fractions produced in the absence of urea. A continuous increase of the amount of MCPs was observed with increasing concentrations of the denaturant. The extent of missed cleavages was lower in the $\mathrm{CaCo}-2$ and muscle than in the brain samples. 

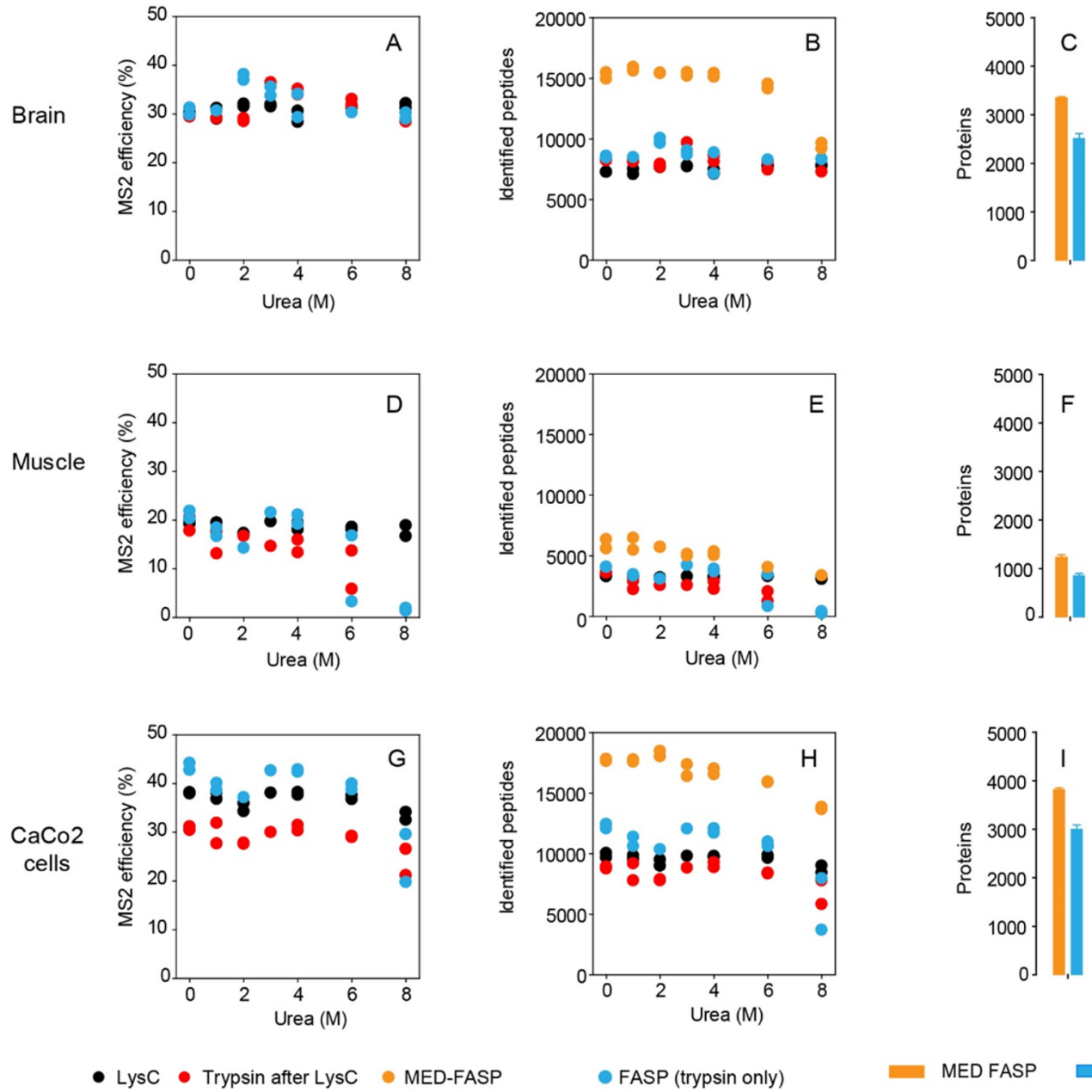

- LysC - Trypsin after LysC • MED-FASP

- FASP (trypsin only)

MEDFASP $\square$ FASP

Figure 3. Identification of unique peptides and proteins by LC-MS/MS using different digestion conditions. Peptides generated by FASP and MED FASP from whole tissue (A-F) and cell (G-I) lysates were separated by $4 \mathrm{~h} \mathrm{LC}$ and analyzed with an Orbitrap instrument. "MS2 efficiency" indicates the portion of MS1 spectra resulting in MS2 spectra leading to peptide identification. The "LysC" (black) and "Trypsin after LysC" (red) symbols refer to MED FASP digestion steps. Bars in C, F, and I show the number of identified proteins per two LC-MS/MS, of either tryptic peptide fractions or single MED FASP composed of a LysC and tryptic digests. The bars are averages of data resulting from the analysis of samples digested in 0,1 , and $2 \mathrm{M}$ urea.

In the FASP procure with trypsin, the amounts of MCPs were several-fold higher compared to the MED FASP. The lowest rates of MCPs were found under digestion in the absence of urea ranging from $26 \%$ for the brain and $\mathrm{CaCo}-2$ samples (Figure 4A,B) to 35\% for muscle lysates (Figure 4C). The presence of the denaturant led to a less efficient digestion and produced higher amounts of MCPs.

FASP Protocols Outperform "In-Solution" Digestion. In the past, several reports questioned the effectiveness of FASP and suggested other digestion strategies as better solutions for sample preparation. . We compared FASP and MED FASP with an "in-solution" digestion method. In this comparison, we used either whole brain lysates or microsomal fractions from mouse liver. We found that FASP resulted in 1.5-2 times more peptides than the "in-solution" protocol (Figure 5A,B). As a consequence of weaker cleavage efficiency, the "in-solution" digests contained about 2-fold more MCPs (Figure 5C). This comparison shows that the FASP methods outperform the digestion "in-solution". Notably, a high content of MCPs in digests prepared by "in-solution" methods was also reported previously $^{23}$. $^{24}$
High Sample to Sample Reproducibility of FASP. Reproducibility of the sample preparation method is a prerequisite for relative and absolute protein quantification. To evaluate reproducibility of the MED FASP and FASP methods, we compared the concentrations of proteins calculated by TPA between single technical repeats using the Pearson Correlation. We compared concentrations of proteins quantified in all samples, which were identified with at least 3 peptides. The set of brain lysate analyses comprised 2957 and 1264 proteins for the MED FASP and FASP experiments, respectively (Figure 6A,B). For all technical repeats, we found an excellent correlation of $r \geq 0.99$ between MED FASP runs and $\geq 0.98$ between FASP single measurements (Figure 6A,B). Comparison of the data across samples processed at various urea concentrations also showed negligible variation $(r \geq 0.97)$ indicating that moderate changes in the denaturant concentrations have practically no effect on the final results. We also analyzed our data using two other label free quantitation (LFQ) approaches, which are used only for relative protein quantitation, the intensity based MaxQuant algorithm, and spectral counting. Whereas the MQ-LFQ data showed similar correlations compared to TPA, the correlations between the 

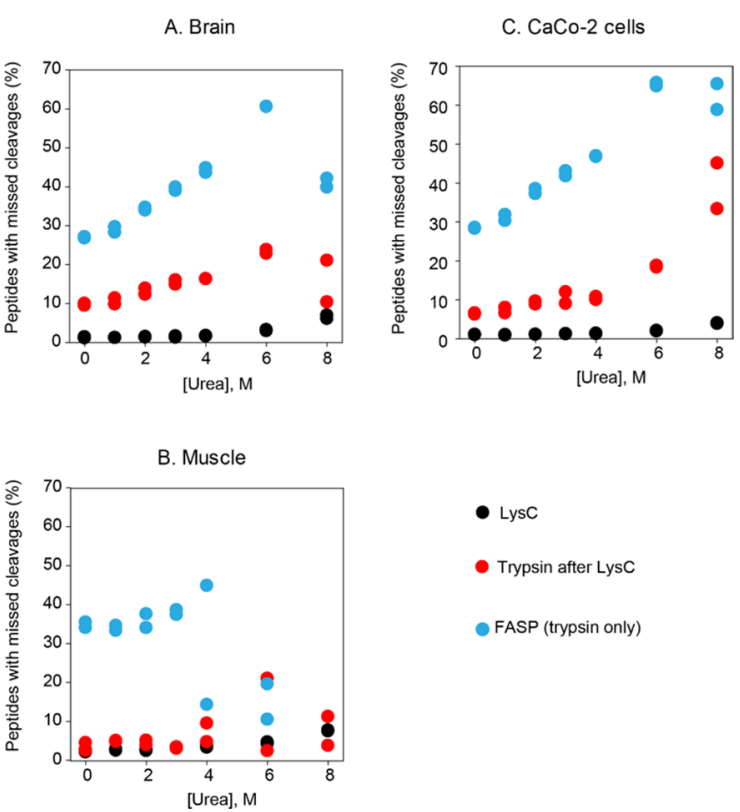

Figure 4. Frequency of identification of peptides with missed cleavages (MCPs). Peptides generated by FASP and MED FASP from brain (A), muscle (B), and CaCo cells (C) lysates were separated by $4 \mathrm{~h} \mathrm{LC}$ and analyzed with an Orbitrap instrument. MaxQuant analysis assessed the numbers of MCPs.
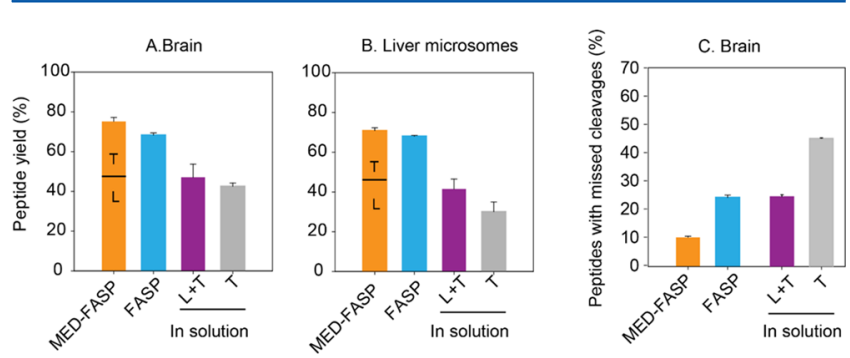

Figure 5. Comparison of peptide yields (A and B) and rates of peptides with missed cleavages (C) observed in samples processed by FASP and "in-solution" methods. Horizontal line in the MED FASP bars indicates the portion of tryptic (T) and LysC (L) peptides contributing to the total peptide yield. After the "in-solution" digestion, any undigested material was removed by ultrafiltration before peptide quantitation and mass spectrometric analysis.

data obtained by a spectral counting approach were more variable (Figure 6C,D). Similarly, a high correlation between single analyses of MED FASP was observed for muscle and CaCo-2 cells (Figure 6E,F). These results clearly demonstrate the robustness of FASP methods for sample processing.

\section{CONCLUSIONS}

In this study, we re-evaluated the FASP and MED FASP procedures using "Microcon" ultrafiltration units. Our results reveal the performance of the methods for the analysis of tissue and cultured cells. We determined the limits in protein amount that can be processed and found that digestion in the absence of urea results in peptide mixtures with the lowest rate of MCPs. The presented results indicate that the sample preparation outcome varies between different sample types. In terms of protein to peptide conversion and missed cleavage rates, FASP outperforms the "in-solution" digestion procedure.

Our results also emphasize advantages of MED FASP over the single enzyme digestion in terms of quality and depth of
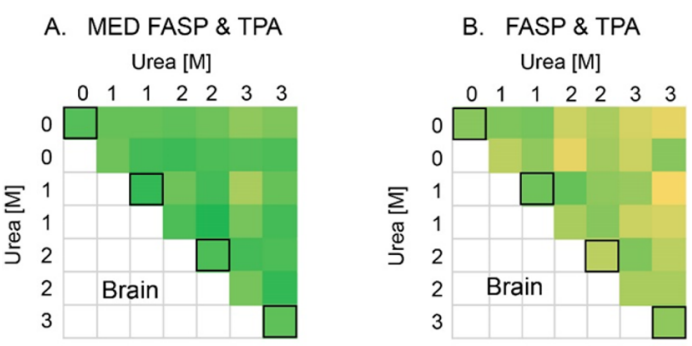

\section{MED FASP \& MQ-LFQ}

\section{MED FASP \& SPC}

Urea [M]
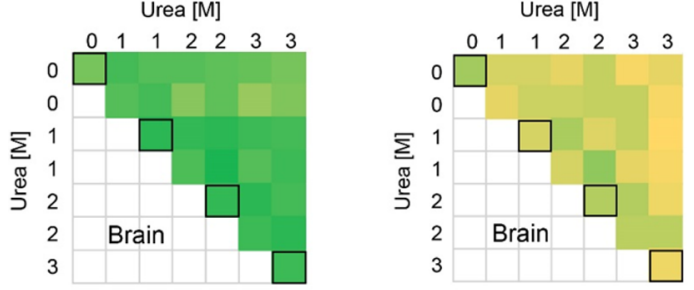

E. MED FASP \& TPA Urea [M]

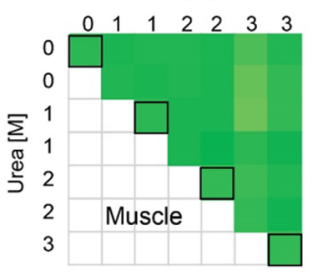

F. MED FASP \& TPA Urea [M]

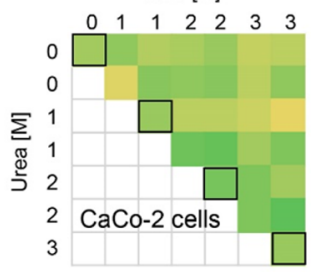

Pearson correlation $(r)$

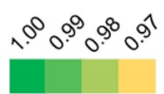

Figure 6. Reproducibility of the MED FASP (A and C-F) and FASP (B) methods. The figure shows the Person Correlation coefficients between the values obtained from the MaxQuant "raw intensity" output processed with the Total Protein Approach (TPA; panels A, B, $\mathrm{E}$, and F), the MaxQuant-"LFQ algorithm" (LFQi panel C) data, and MaxQuant-"spectral counts" (SPC; panel D) for brain (A-D), muscle (E), and $\mathrm{CaCo}-2$ lysates (F). Only proteins identified in all single analyses with at least 3 peptides were selected. The data cover duplicate analyses of samples digested in the presence of $0,1,2$, or 3 $\mathrm{M}$ urea. The technical duplicates are boxed.

proteomic analysis. It has been well documented that elution of peptides after the first digestion changes the cleavage reaction equilibrium, which in-turn facilitates digestion of less abundant proteins as well as digestion of sites with lower affinity to enzyme. ${ }^{11,25}$ Moreover, reduction of the peptide concentrations by stepwise elution and digestion may reduce an often unnoticed process of transpeptidation, in which already released peptides and amino acids are transferred to another amino compound. ${ }^{26}$ Obviously, transpeptidation products escape detection during common data searches.

In this study, we focus on FASP methods applied for total protein amounts larger than $25 \mu \mathrm{g}$. We expect that our conclusions are also valid for preparations using smaller sample amounts. For example, in a recently reported application of FASP to low protein level samples, as the secretome from murine Langerhans islets, digestion was conducted in the absence of any denaturant. ${ }^{27}$ This is in line with the findings of 
this work showing that urea worsens rather than improves the performance of protein digestion.

In conclusion, evaluation of the FASP protocols, using the "Microcon" ultrafiltration units, demonstrates that the methods give excellent performance for samples between 25 and $100 \mu \mathrm{g}$ of total protein. For the highest protein to peptide conversion and the lowest rate of peptides with missed cleavages, protein digestion have to be carried out in the absence of urea using MED FASP.

\section{ASSOCIATED CONTENT}

\section{S Supporting Information}

The Supporting Information is available free of charge on the ACS Publications website at DOI: 10.1021/acs.analchem.6b00859.

Supplementary Table 1, peptides identified by the analysis of mouse brain lysates (XLSX)

Supplementary Table 2, peptides identified by the analysis of mouse muscle lysates. (XLSX)

Supplementary Table 3, peptides identified by the analysis of human $\mathrm{CaCo}-2$ cells. (XLSX)

Supplementary Table 4, proteins identified by the analysis of mouse tissue and $\mathrm{CaCo}-2$ cell lysates. (XLSX)

Supplementary Table 5, peptides identified by the analysis of mouse brain lysates using an "in-solution" digestion method. (XLSX)

\section{AUTHOR INFORMATION}

\section{Corresponding Author}

*Tel.: +49898578 2205. E-mail: jwisniew@biochem.mpg.de.

Notes

The authors declare no competing financial interest.

\section{ACKNOWLEDGMENTS}

We thank Prof. Matthias Mann for continuous support, Dr. Georg Borner for critical reading of the manuscript, Katharina Zettl for technical assistance, and Igor Paron and Korbinian Mayr for support in mass spectrometric analysis. This work was supported by the Max Planck Society for the Advancement of Science and the German Research Foundation (DFG/Gottfried Wilhelm Leibniz Prize). The mass spectrometry proteomics data have been deposited to the ProteomeXchange Consortium $^{28}$ via the PRIDE partner repository with the data set PXD003733.

\section{REFERENCES}

(1) Kulak, N. A.; Pichler, G.; Paron, I.; Nagaraj, N.; Mann, M. Nat. Methods 2014, 11, 319-324.

(2) Hughes, C. S.; Foehr, S.; Garfield, D. A.; Furlong, E. E.; Steinmetz, L. M.; Krijgsveld, J. Mol. Syst. Biol. 2014, 10, 757.

(3) Ethier, M.; Hou, W.; Duewel, H. S.; Figeys, D. J. Proteome Res. 2006, 5, 2754-2759.

(4) Manza, L. L.; Stamer, S. L.; Ham, A. J.; Codreanu, S. G.; Liebler, D. C. Proteomics 2005, 5, 1742-1745.

(5) Wisniewski, J. R.; Zougman, A.; Nagaraj, N.; Mann, M. Nat. Methods 2009, 6, 359-362.

(6) Crowell, A. M.; MacLellan, D. L.; Doucette, A. A. J. Proteomics 2015, 118, 140-150.

(7) Fischer, R.; Kessler, B. M. Proteomics 2015, 15, 1224-1229.

(8) Zougman, A.; Selby, P. J.; Banks, R. E. Proteomics 2014, 14, $1006-1000$.
(9) Zhang, Z.; Sun, L.; Zhu, G.; Cox, O. F.; Huber, P. W.; Dovichi, N. J. Anal. Chem. 2016, 88, 877-882.

(10) Wisniewski, J. R.; Mann, M. Anal. Chem. 2012, 84, 2631-2637.

(11) Fonslow, B. R.; Stein, B. D.; Webb, K. J.; Xu, T.; Choi, J.; Park,

S. K.; Yates, J. R., 3rd Nat. Methods 2013, 10, 54-56.

(12) Wisniewski, J. R.; Prus, G. Anal. Chem. 2015, 87, 6861-6867.

(13) Washburn, M. P.; Wolters, D.; Yates, J. R., 3rd Nat. Biotechnol. 2001, 19, 242-247.

(14) Wisniewski, J. R.; Rakus, D. J. Proteomics 2014, 109, 322-331.

(15) Wisniewski, J. R. J. Vis. Exp. 2013, No. 79, 50589.

(16) Wisniewski, J. R.; Ostasiewicz, P.; Mann, M. J.Proteome Res. 2011, 10, 3040-3049.

(17) Ishihama, Y.; Rappsilber, J.; Andersen, J. S.; Mann, M. J.Chromatogr. A 2002, 979, 233-239.

(18) Wisniewski, J. R.; Gaugaz, F. Z. Anal. Chem. 2015, 87, 41104116.

(19) Wisniewski, J. R.; Koepsell, H.; Gizak, A.; Rakus, D. Proteomics 2015, 15, 1316-1325.

(20) Wisniewski, J. R.; Mann, M. Anal. Chem. 2012, 84, 2631-2637.

(21) Cox, J.; Mann, M. Nat. Biotechnol. 2008, 26, 1367-1372.

(22) Wisniewski, J. R.; Zielinska, D. F.; Mann, M. Anal. Biochem. 2011, 410, 307-309.

(23) Glatter, T.; Ludwig, C.; Ahrne, E.; Aebersold, R.; Heck, A. J.; Schmidt, A. J. Proteome Res. 2012, 11, 5145-5156.

(24) Chiva, C.; Ortega, M.; Sabido, E. J. Proteome Res. 2014, 13, 3979-3986.

(25) Fonslow, B. R.; Stein, B. D.; Webb, K. J.; Xu, T.; Choi, J.; Park, S. K.; Yates, J. R., 3rd Nat. Methods 2014, 11, 347-348.

(26) Schaefer, H.; Chamrad, D. C.; Marcus, K.; Reidegeld, K. A.; Bluggel, M.; Meyer, H. E. Proteomics 2005, 5, 846-852.

(27) Schmudlach, A.; Felton, J.; Cipolla, C.; Sun, L.; Kennedy, R. T.; Dovichi, N. J. Analyst 2016, 141, 1700-1706.

(28) Vizcaino, J. A.; Deutsch, E. W.; Wang, R.; Csordas, A.; Reisinger, F.; Rios, D.; Dianes, J. A.; Sun, Z.; Farrah, T.; Bandeira, N.; Binz, P. A.; Xenarios, I.; Eisenacher, M.; Mayer, G.; Gatto, L.; Campos, A.; Chalkley, R. J.; Kraus, H. J.; Albar, J. P.; Martinez-Bartolome, S.; Apweiler, R.; Omenn, G. S.; Martens, L.; Jones, A. R.; Hermjakob, H. Nat. Biotechnol. 2014, 32, 223-226. 